\title{
Hund-Heisenberg model in superconducting infinite-layer nickelates
}

\author{
Jun Chang ${ }^{1}$, Jize Zhao ${ }^{2}$, Yang Ding ${ }^{3}$ \\ ${ }^{1}$ College of Physics and Information Technology, \\ Shaanxi Normal University, Xi'an 710119, China \\ ${ }^{2}$ School of Physical Science and Technology\&Key Laboratory for Magnetism and \\ Magnetic Materials of the MoE, Lanzhou University, Lanzhou 730000, China \\ ${ }^{3}$ Center for High-Pressure Science and Technology Advanced Research, Beijing 100094, China
}

(Dated: October 15, 2020)

\begin{abstract}
We theoretically investigate the unconventional superconductivity in the newly discovered infinitelayer nickelates $\mathrm{Nd}_{1-x} \mathrm{Sr}_{x} \mathrm{NiO}_{2}$ based on a two-band model. By analyzing the transport experiments, we propose that the doped holes dominantly enter the $\mathrm{Ni} d_{x y}$ or/and $d_{3 z^{2}-r^{2}}$ orbitals as charged carriers, and form a conducting band. Via the onsite Hund coupling, the doped holes are coupled to the $\mathrm{Ni}$ localized holes in the $d_{x^{2}-y^{2}}$ orbital band. We demonstrate that this two-band model could be further reduced to a Hund-Heisenberg model. Using the reduced model, we show the non-Fermi liquid state above the critical $T_{c}$ could stem from the carriers coupled to the spin fluctuations of the localized holes. In the superconducting phase, the short-range spin fluctuations mediate the carriers into Cooper pairs and establish $d_{x^{2}-y^{2}}$-wave superconductivity. We further predict that the doped holes ferromagnetically coupled with the local magnetic moments remain itinerant even at very low temperature, and thus the pseudogap hardly emerges in nickelates. Our work provides a new superconductivity mechanism for strongly correlated multi-orbital systems and paves a distinct way to exploring new superconductors in transition or rare-earth metal oxides.
\end{abstract}

\section{INTRODUCTION}

Various unconventional superconductors have proliferated experimentally over the recent decades although the origins of the superconductivity (SC) in cuprates and heavy fermion materials remain theoretically controversial [1]. The quasi-two-dimensional (2D) iron pnictides have triggered a new boom of the SC investigation [4. In particular, the search efforts for the compounds with the geometrical and electronic structure similar to cuprates are growing both experimentally and theoretically due to the highest critical temperatures at ambient conditions. Isostructural compounds, e.g. $\mathrm{Sr}_{2} \mathrm{RuO}_{4}, \mathrm{Sr}_{2} \mathrm{IrO}_{4}$ and $\mathrm{LaNiO}_{2}$ as well as some artificial heterostructure have been extensively proposed, and synthetized $5+20$. The unremitting pursuits have been rewarded despite no evidence of SC in some of these analogs so far.

Recently, the exciting discovery of superconductivity in the hole doped infinite-layer nickelate $\mathrm{Nd}_{1-x} \mathrm{Sr}_{x} \mathrm{NiO}_{2}$ redraws strong attention to the unconventional SC 6, 2125. The quasi-2D Ni-O plane is geometrically analog to the $\mathrm{Cu}-\mathrm{O}$ plane in cuprates. The $d_{x^{2}-y^{2}}$ orbital of each $\mathrm{Ni}^{1+}$ ion is also half-filled, with an effective spin- $1 / 2$ on each site. However, the differences from cuprates are notably striking. In the parent compounds, there is no sign of long-range magnetic orders in the measured temperature range [26. Maybe due to self-doping effects, the electrons of the rare-earth $\mathrm{Nd}$ between $\mathrm{Ni}-\mathrm{O}$ planes form a 3D weakly-interacting $5 d$ metallic state with an electronic Fermi surface [21, 27, 29. Intriguingly, the resistivity exhibits metallic temperature dependence down to $60 \mathrm{~K}$, and then shows insulating upturn at lower temperatures, which could be the results of weak localization effects, Kondo effects or temperature driven intra-band transitions [6, 30, 31]. Upon chemical doping, additional holes dominantly enter the $d$ orbitals of the Ni ions rather than $\mathrm{O}$ orbitals as in cuprates since the $\mathrm{O} 2 p$ states are far away from the Fermi level in nickelates [8, 23, 27, 32, 33. The sign change of the Hall coefficient at low temperature indicates that both electrons and holes may contribute to the transport and thermodynamic properties [6. Moreover, it is debating whether the doped hole forms a spin singlet or triplet doublon with the original hole on a Ni ion 3236 . Several microscopic models have been proposed, such as the $t-J$ models, the metallic gas coupled to a 2D Hubbard model and the spin freezing model [21, 27, 28, 33 37. More surprisingly, absence of superconductivity was recently claimed in the bulk nickelates and the film prepared on various oxide substrates different from $\mathrm{SrTiO}$. It was suggested that the absence possibly results from the hydrogen intercalation [38 40]. Own to these confusions, more insights into the microscopic mechanism in nickelates are imperative.

In this paper, we investigate the nickelate $\mathrm{SC}$ based on the analysis of the transport experiments. Considering the positive Hall coefficient and the suppressed selfdoping effects at low temperature, we suggest that since the Ni $d_{x y}$ or/and $d_{3 z^{2}-r^{2}}$ orbital is close to Fermi energy level, the doped holes may go to these orbitals and establish a conducting band 8, 22, 23, 41. The onsite Hund interaction couples the conducting band with the localized $d_{x^{2}-y^{2}}$ orbital band together. The correlation between the sparse carriers and the kinetic energy of the localized holes could then be ignored. Thus, the twoband model is simplified into a Hund-Heisenberg model. We show that both the non-Fermi liquid in normal state and the superconductivity is determined by the spin fluctuations of the localized holes. This SC mechanism could be realized in multi-orbital strongly correlated systems with both Hund and Heisenberg interactions [42 45]. 


\section{MICROSCOPIC HAMILTONIAN}

We first analyze the electronic properties in normal state based on the transport experiments [6]. In the parent compounds, both the resistivity and Hall effect measurements show Kondo effects with logarithmic temperature dependence from tens to around several kelvin. The Kondo effects were attributed to the hybridization between the $\mathrm{Nd} 5 d$ states and the $\mathrm{Nd} 4 f$ or $\mathrm{Ni} 3 d$ states as in rare-earth heavy fermion compounds although the $a b$ initio study suggests the hybridization between the $\mathrm{Ni} 3 d$ state and the $\mathrm{Nd} 5 d$ states is negligible, and the If electron spin fluctuation should be weak due to the large magnetic moment and the energy far away from Fermi energy level [27-29, 36]. In $\mathrm{Nd}_{0.8} \mathrm{Sr}_{0.2} \mathrm{NiO}_{2}$, above $60 \mathrm{~K}$, the negative Hall coefficient indicates that the $\mathrm{Nd}$ $5 d$ electrons dominate the transport and thermodynamic properties. With the decreasing of temperature, the selfdoping effect is reduced as in semiconductors and the Hall coefficient also changes its sign from negative to positive. This means that the doped holes take over the dominant role in the transport and thermodynamics at low temperature. In addition, the Hund coupling between the $3 d$ doped holes and localized holes is around an order of magnitude stronger than the Kondo coupling between the $5 d$ electron and the $3 d$ magnetic moments. Therefore, we ignore the $5 d$ electrons in our model, and in our discussion section we show that they only give negligible contribution to the superconductivity and non-Fermiliquid behavior in the normal state. Whether the doped hole forms a high spin triplet or a low spin singlet doublon with the original hole on the $d_{x^{2}-y^{2}}$ orbital is still controversial 3236 . In fact, a $\mathrm{Ni}^{2+}$ ion with $d^{8}$ configuration often has a high spin $\mathrm{S}=1$ in common Nickel oxides as the result of Hund coupling. According to the first principle calculation, the energy of the triplet state is around $1 \mathrm{eV}$ lower than that of the singlet, and the top of the Ni $d_{x y}$ or/and $d_{3 z^{2}-r^{2}}$ orbital band is also close to the Fermi energy [8, 22, 23, 41. Moreover, the holes doped on the $d_{x y}$ or/and $d_{3 z^{2}-r^{2}}$ orbitals could itinerate freely, agreeing well with the positive Hall coefficient at low temperature. The delocalization of the doped holes on $d_{x y}$ or/and $d_{3 z^{2}-r^{2}}$ orbitals is attributed to the fact that the doped hole concentration is dilute, and under the short range antiferromagetic (AF) correlation background, a hole can hop freely to its next nearest neighbor sites without energy cost as long as these sites are not occupied by another doped hole. In contrast, the doped holes on the already half-filled $d_{x^{2}-y^{2}}$ orbitals tend to be localized at low temperature, otherwise the hopping disturbs the magnetic configurations of short-range AF correlations as in cuprates [3].

Based on the aforementioned analysis, we confine our study to the Ni-O planes and assume that the holes doped on the $d_{x y}$ or/and $d_{3 z^{2}-r^{2}}$ orbitals form a conducting band, coexisting with the localized $d_{x^{2}-y^{2}}$ orbital band. $c_{i}=\left(c_{i \uparrow}, c_{i \downarrow}\right)^{T}$ is introduced as the annihilation operator of the bare carrier particles on the $d_{x y}$ or/and $d_{3 z^{2}-r^{2}}$

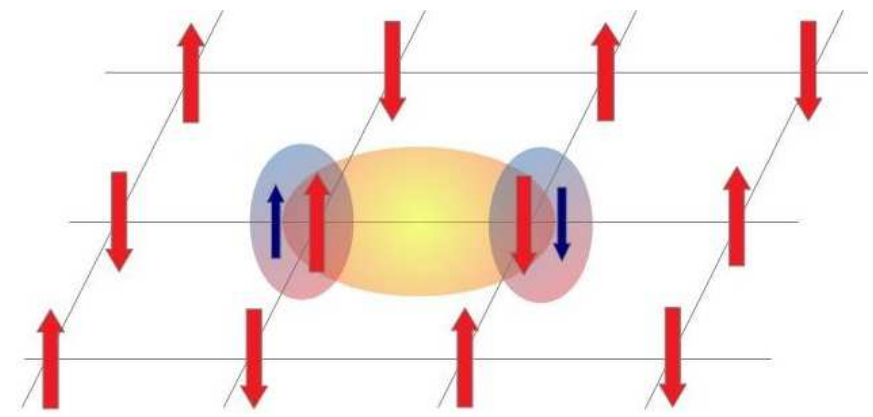

FIG. 1: Schematic spin configuration on the Ni-O planes of the hole-doped nickenates. The red thicker arrows denote the spins of the localized holes in the $d_{x^{2}-y^{2}}$ orbital band. The local magnetic moments interact with each other through the Heisenberg interaction. The blue arrows denotes the spins of the doped holes as carriers in the conducting $d_{x y}$ or/and $d_{3 z^{2}-r^{2}}$ orbital band. Their spins are parallel with the local magnetic moments due to the strong Hund coupling. In normal state, the scattering by the spin fluctuations of the localized holes transfers the carrier Fermi gas into a non-Fermi liquid. A carrier could hop to its next nearest neighbor sites without any energy cost as long as these sites are not occupied by other doped holes. Thus the doped holes itinerate over the lattice even at very low temperature without formation of a pseudogap. While in superconducting phase, two neighboring carrier particles are mediated into a Cooper pair by the spin fluctuations of the local magnetic moments.

orbitals of the $i$ th $\mathrm{Ni}$ site, and $d_{i}=\left(d_{i \uparrow}, d_{i \downarrow}\right)^{T}$ is the real space annihilation operator of the localized holes on the Ni $d_{x^{2}-y^{2}}$ orbitals. The Hamiltonian is written as

$$
\begin{aligned}
H= & H_{c}+H_{d}+U_{c} \sum_{i} n_{c i \uparrow} n_{c i \downarrow}+U_{d} \sum_{i} n_{d i \uparrow} n_{d i \downarrow} \\
& +U_{c d} \sum_{i} n_{c i} n_{d i}-J_{h} \sum_{i} \mathbf{S}_{c i} \cdot \mathbf{S}_{d i}
\end{aligned}
$$

with

$$
H_{c}=\varepsilon_{c 0} \sum_{i} c_{i}^{\dagger} c_{i}-\sum_{i, j} t_{c i j} c_{i}^{\dagger} c_{j}
$$

and

$$
H_{d}=\varepsilon_{d 0} \sum_{i} d_{i}^{\dagger} d_{i}-\sum_{i, j} t_{d i j} d_{i}^{\dagger} d_{j}
$$

where $t_{c i j}$ and $t_{d i j}$ are the hopping integrals of the carriers in conducting band and the localized holes in $d_{x^{2}-y^{2}}$ band, respectively. $n_{c i}=c_{i}^{\dagger} c_{i}=n_{c i \uparrow}+n_{c i \downarrow}$ is the occupancy of the carriers with spin up and down on the $i$ th site, and $n_{d i}$ is the occupancy of the $d_{x^{2}-y^{2}}$ orbital. $J_{h}$ is the Hund coupling between the carriers and the localized holes on the same site. $U_{c}$ is the onsite Coulomb repulsion between carriers, $U_{d}$ is the interaction between the localized holes, and $U_{c d}$ is the inter-orbital Coulomb repulsion between the carrier and localized hole on the same site. 
To further simplify the Hamiltonian, based on the fact that the hopping term $t_{d i j}$ is much less than the large onsite Coulomb interaction $U_{d}$, we take the $t_{d i j}$ as the perturbation then the Hubbard model for the localized $d_{x^{2}-y^{2}}$ orbital band is reduced to a Heisenberg model. In addition, the large onsite Coulomb interaction strongly suppresses the particle number fluctuation on the halffilled $d_{x^{2}-y^{2}}$ orbitals, so that the Coulomb interaction acting on the carriers by the localized holes could be renormalized into the chemical potential within the singly occupied approximation $\left\langle n_{d i}\right\rangle=1$. Furthermore, in consideration of the delocalization and the sparse concentration $\left\langle n_{c i}\right\rangle$ of the doped holes in conducting band, we can safely ignore the correlation between the carriers as in common metals. Alternatively, the magnetic coupling $\mathbf{S}_{c i} \cdot \mathbf{S}_{c j}$ term is ignored due to the much weaker magnetization of the carriers than that of the localized holes. Finally, we arrive at a Hund-Heisenberg model,

$$
H=H_{c}-J_{h} \sum_{i} \mathbf{S}_{c i} \cdot \mathbf{S}_{d i}+J_{H} \sum_{\langle i, j\rangle} \mathbf{S}_{d i} \cdot \mathbf{S}_{d j}
$$

where $\mathbf{S}_{c i}=c_{i}^{\dagger} \boldsymbol{\sigma} c_{i} / 2$ and $\mathbf{S}_{d i}=d_{i}^{\dagger} \boldsymbol{\sigma} d_{i} / 2$ with the Pauli vector $\boldsymbol{\sigma} . J_{H}$ is the Heisenberg interaction between the $d_{x^{2}-y^{2}}$ orbital holes on the Ni square lattice. Here, we have ignored the kinetic energy of the localized holes, and the carrier chemical potential $\varepsilon_{c 0}$ has been replaced by $\varepsilon_{c}=\varepsilon_{c 0}+U_{c d}$ in $H_{c}$ to include the energy renormalization from the Coulomb interaction of the holes on the $d_{x^{2}-y^{2}}$ orbitals.

This model is formally similar to the KondoHeisenberg model on a 2D square lattice [46, 47]. The difference is the conducting carrier ferromagnetically coupled to the localized magnetic moments rather than antiferromangetically. In addition, the onsite Hund ferromagnetic coupling is in favor of the delocalization of the doped holes under the antiferromagnetic background even at very low temperature, and thus it is difficult to form a pseudogap. In the following, we show that the spin fluctuations of the localized $d_{x^{2}-y^{2}}$ states not only act as the pairing 'glue' in superconducting state, but also results in non-Fermi liquid in normal state.

\section{NORMAL STATE}

The bare doped holes are assumed to compose a dilute Fermi gas with the retarded Green's function $G_{c}^{0}(\mathbf{k}, \omega)$, bathed in the Heisenberg antiferromagnets of the localized holes. At low temperature, the transport and thermodynamic properties are determined by the imaginary part of the carrier self-energy. However, different from the Fermi liquids, the dominant contribution to the selfenergy is from the interaction between the carriers and the localized holes rather than the carrier-carrier interaction. Because the concentration of the carriers is much lower than the localized holes despite the same order strength of the two kinds of interactions. Therefore, we ignore the self-energy correction by the carrier-carrier correlation interaction. Within the Born approximation, the imaginary part of the self-energy correction by the renormalized spin fluctuation $\chi_{d}(\mathbf{q}, \omega)$ of the localized $d_{x^{2}-y^{2}}$ holes reads 46 ]

$$
\operatorname{Im} \Sigma_{c}(\mathbf{k}, \omega) \sim J_{h}^{2} \int_{-\omega_{c}}^{\omega_{c}} d v\left[n_{B}(v)+n_{F}(\omega+v)\right] I(\mathbf{k}, \omega, v),
$$

where $n_{B}$ and $n_{F}$ are the Bose and Fermi functions, $\omega_{c}$ is the upper cutoff frequency of magnetic fluctuations and

$$
I(\mathbf{k}, \omega, v)=\int \frac{d^{2} q}{4 \pi^{2}} \operatorname{Im} \chi_{d}(\mathbf{q}, v) \operatorname{Im} G_{c}^{0}(\mathbf{k}+\mathbf{q}, \omega+v) .
$$

It is worth noting that the neglect of the higher order self-energy corrections is based on the fact that the carriers is weakly magnetized by the local magnetic moments or $\left|\left\langle\mathbf{S}_{c i}\right\rangle\right| \ll 1 / 2$. Therefore, the Hund coupling in the effective Hamiltonian only gives perturbation correction to the carrier self-energy despite the large Hund coupling constant in Eq. (4). However, the the approximation of neglecting the higher order corrections may be questionable if the $\operatorname{Im} \chi_{d}(\mathbf{q}, v) \sim v^{s}$ with $s \leq 0$ at low frequency. For instance, the perturbation related coupling constant $\lambda_{\mathbf{q}}=2 \int_{0}^{\infty} d v \alpha_{\mathbf{q}}^{2}(v) \operatorname{Im} \chi_{d}(\mathbf{q}, v) / v$ diverges, where $\alpha_{\mathbf{q}}^{2}(v) \operatorname{Im} \chi_{d}(\mathbf{q}, v)$ is the generalized McMillan carrier-boson coupling function.

Integrating over the momentum $\mathbf{k}$, one finds the momentum-integral imaginary part of the Fermi gas selfenergy

$$
\operatorname{Im} \Sigma_{c}(\omega) \sim J_{h}^{2} \int_{-\omega_{c}}^{\omega_{c}} d v\left[n_{B}(v)+n_{F}(\omega+v)\right] \operatorname{Im} \chi_{d}(v) \rho_{c}(\omega+v)
$$

with the aid of

$$
\int \frac{d^{2} k}{4 \pi^{2}} I(\mathbf{k}, \omega, v)=-\pi \rho_{c}(\omega+v) \operatorname{Im} \chi_{d}(v)
$$

where the magnetic fluctuations $\chi_{d}(v) \equiv$ $\int d^{2} q \chi_{d}(\mathbf{q}, v) / 4 \pi^{2} . \quad \rho_{c}$ is the density of states of the carriers. Since the absolute value of $n_{B}(v)+n_{F}(\omega+v)$ exponentially decreases to zero with increasing $|v|$, the energy range of integration can be extended from the cutoff $\omega_{c}$ to infinity, and $\rho_{c}(\omega+v)$ is approximately substituted by $\rho_{c}^{0}$, the carrier density of states at Fermi energy level. One has

$$
\operatorname{Im} \Sigma_{c}(\omega) \sim J_{h}^{2} \rho_{c}^{0} \int_{-\infty}^{\infty} d v\left[n_{B}(v)+n_{F}(\omega+v)\right] \operatorname{Im} \chi_{d}(v)
$$

Since no experimental or theoretical results on $\operatorname{Im} \chi_{d}(v)$ could be obtained presently we assume that the momentum-integral spin-fluctuation spectra of the halffilled $d_{x^{2}-y^{2}}$ holes take the similar form of the underdoped cuprates 48 50, e.g. $\operatorname{Im} \chi_{d}(v) \sim \tanh (v / 2 T)$. Using the analytical frequency integral equation 46 . 


$$
\begin{array}{r}
\int_{-\infty}^{\infty} d v\left[n_{B}(v)+n_{F}(\omega+v)\right] \tanh \left(\frac{v}{2 T}\right) \\
=2 T\left[1+\frac{\omega}{2 T} \tanh \left(\frac{\omega}{2 T}\right)\right],
\end{array}
$$

the carriers have the marginal Fermi liquid-like selfenergy

$$
\begin{aligned}
\operatorname{Im} \Sigma_{c}(\omega, T) & \sim \pi \rho_{c}^{0} J_{h}^{2} T\left[1+\frac{\omega}{2 T} \tanh \left(\frac{\omega}{2 T}\right)\right] \\
& \sim \max (|\omega|, T) .
\end{aligned}
$$

Then the linear temperature dependence of electrical resistivity observed in the experiments could be explained 6], and some other anomalous transport properties are expected to be experimentally verified.

In cuprates, since the doped holes are antiferromagnetically coupled to the localized holes, the hopping disturbs the original magnetic configuration, and thus the doped holes tend to be localized at low doping or at low temperature 3. On the contrary, in nickelates, the doped holes, which are ferromagnetically coupled to localized holes, could hop over the Ni-O plane without affecting the magnetic background so that they are not easy to be trapped around the local magnetic moments even at low temperature. In addition, a carrier could hop to its next nearest neighbor sites without any energy cost as long as these sites are not occupied by other doped holes. Therefore, we propose that it is almost impossible to observe a pseudogap in nickelates.

\section{SUPERCONDUCTIVITY}

In normal state, the carriers are scattered by the shortrange spin fluctuation as the metallic gas by phonons. In the superconducting state, the carrier pairing is mediated by the spin fluctuations analogues of the pairing by phonons in the BCS mechanism. It is worthy of note that we assume that the conducting carriers only partially screen the local moments without formation of localized triplets or singlets, and then the Heisenberg interaction between the screened moments and their surroundings could survive. Thus, the carriers on unit cell $i$ and $j$ could interact with each other by exchange of the spin fluctuation in terms of a four point vertex, written in real space as

$$
\Gamma_{\alpha \beta, \gamma \delta}(i, j, \omega)=-\frac{J_{h}^{2}}{4} \chi_{d}(i, j, \omega) \sigma_{\alpha \beta} \sigma_{\gamma \delta} .
$$

We assume that the AF spin fluctuation only mediates the itinerant holes spacing within the AF correlation length into stable Cooper pairs. The correlation length in nickelates is assumed to be the same scale as that in cuprates at low temperature, around two times the lattice constant. Thus, we only take the nearest-neighbor $\chi_{d}(\langle i, j\rangle, \omega)$ into account. Then, the interaction Hamiltonian of the carriers can be written in the coordinate representation as 46

$$
H_{s c}=J_{h}^{2} \chi_{d}(\langle i, j\rangle, \omega) \sum_{\langle i, j\rangle} \mathbf{S}_{c i} \cdot \mathbf{S}_{c j},
$$

where the nearest neighbor $\chi_{d}(\langle i, j\rangle, \omega)$ is assumed to be space independent. For a local pair, the energy of a spin-triplet is about $J_{h}^{2} \chi_{d}(\langle i, j\rangle, \omega)$ higher than that of a spin-singlet, and thus the antiferromagnetic spin fluctuations favors spin-singlet pairing. Combining with $H_{c}$ in Eq. 2), a $t$ - $J$-like model is reached. Interestingly, despite the formal similarity with the conventional $t-J$ model [51], here the spin-like operator $\mathbf{s}$ is associated with the carriers rather than the localized holes.

After transforming to momentum space, the Hamiltonian on the square lattice becomes

$$
H_{s c}=\int \frac{d^{2} k d^{2} k^{\prime}}{(2 \pi)^{4}} J\left(\mathbf{k}-\mathbf{k}^{\prime}\right) c_{\mathbf{k} \uparrow}^{\dagger} c_{-\mathbf{k} \downarrow}^{\dagger} c_{-\mathbf{k}^{\prime} \downarrow} c_{\mathbf{k}^{\prime} \uparrow},
$$

with

$$
J\left(\mathbf{k}-\mathbf{k}^{\prime}\right)=-2 g\left[\cos \left(k_{x}-k_{x}^{\prime}\right)+\cos \left(k_{y}-k_{y}^{\prime}\right)\right]
$$

and the effective coupling between the carriers

$$
g \equiv \frac{3}{4} J_{h}^{2} \chi_{d}(\langle i, j\rangle, \omega)
$$

The Cooper pairing potentials are symmetrized with $J\left(\mathbf{k}-\mathbf{k}^{\prime}\right)$ and $J\left(\mathbf{k}+\mathbf{k}^{\prime}\right)$ in the singlet channel as 52

$$
V_{\mathbf{k}, \mathbf{k}^{\prime}}=\frac{J\left(\mathbf{k}-\mathbf{k}^{\prime}\right)+J\left(\mathbf{k}+\mathbf{k}^{\prime}\right)}{2}
$$

i.e.

$$
V_{\mathbf{k}, \mathbf{k}^{\prime}}=-2 g\left[\cos k_{x} \cos k_{x}^{\prime}+\cos k_{y} \cos k_{y}^{\prime}\right]
$$

The pairing interaction can be further decoupled into $d$-wave and $s$-wave components,

$$
2 \cos k_{x} \cos k_{x}^{\prime}+2 \cos k_{y} \cos k_{y}^{\prime}=\gamma_{k} \gamma_{k^{\prime}}+\gamma_{k}^{s} \gamma_{k^{\prime}}^{s},
$$

with the $d$-wave gap function $\gamma_{k}=\cos k_{x}-\cos k_{y}$, and the extended $s$-wave gap function $\gamma_{k}^{s}=\cos k_{x}+\cos k_{y}$. For $s$-wave superconductivity, the pairing interaction is expected to be negative and nearly isotropic. However, the interaction $V_{\mathbf{k}, \mathbf{k}^{\prime}}$ is positive at $\mathbf{k}-\mathbf{k}^{\prime} \sim \mathbf{Q}$, and the $\mathrm{AF}$ spin fluctuation is strongly momentum dependent, i.e. peaked at or near the AF wave vector $\mathbf{Q}[28$. Therefore, only the $d$-wave pairing channel is favored in the spin- fluctuation SC mechanism [53 61] and the attractive pairing interaction dominantly mediates the carriers on the nearest-neighbor unit cells 62. Thus, the pairing interaction is $V_{\mathbf{k}, \mathbf{k}^{\prime}}^{d}=-g \gamma_{k} \gamma_{k^{\prime}}$ in the $d_{x^{2}-y^{2}}$ channel, which could be detected in phase sensitive interference 
measurements 63. Consequently, in momentum space, a weak coupling BCS interaction can be written

$$
H_{s c d}=-g \int \frac{d^{2} k}{4 \pi^{2}} \gamma_{\mathbf{k}} c_{\mathbf{k} \uparrow}^{\dagger} c_{-\mathbf{k} \downarrow}^{\dagger} \int \frac{d^{2} k^{\prime}}{4 \pi^{2}} \gamma_{\mathbf{k}^{\prime}} c_{\mathbf{k}^{\prime} \uparrow} c_{-\mathbf{k}^{\prime} \downarrow},
$$

The superconductivity order parameter $\gamma_{\mathbf{k}} \Delta_{s c}$ is introduced in the mean field method with the BCS gap equation

$$
\Delta_{s c}=-g \int \frac{d^{2} k}{4 \pi^{2}} \gamma_{\mathbf{k}}<c_{\mathbf{k} \uparrow}^{\dagger} c_{-\mathbf{k} \downarrow}^{\dagger}>,
$$

where $c_{\mathbf{k} \uparrow}^{\dagger} c_{-\mathbf{k} \downarrow}^{\dagger}$ is the Cooper pair operator denoting a bond state of two carriers with opposite momentum and spin.

Solving the gap equation in the limit $\Delta_{s c}\left(T \rightarrow T_{c}\right) \rightarrow$ 0 , the SC transition temperature $T_{c} \sim \omega_{c} e^{-1 / \lambda}$ with $\lambda=g \rho_{c}^{0} / 2$ for weak coupling $d$-wave superconductors. Since the AF correlation in nickelates is weaker than that in cuprates, the spin fluctuation cutoff energy $\hbar \omega_{c} \sim J_{H}$ should be lower than that in cuprates. Moreover, the Hund coupling $J_{h}$ is smaller than the magnetic coupling $J_{K}$ between the $\mathrm{O}$ carriers and $\mathrm{Cu}$ local moments in cuprates. Therefore, the lower critical temperature $T_{c}$ in nickelates could be understood.

\section{DISCUSSION AND CONCLUSION}

Actually, we could not exclude the possibility that the doped holes go to the $\mathrm{Ni} d_{x^{2}-y^{2}}$ orbitals although the strong onsite Coulomb repulsion pushes the $d_{x^{2}-y^{2}}$ lower Hubbard band away from the Fermi level [8, 64]. Nevertheless, if the doped holes forms onsite spin singlet on the $d_{x^{2}-y^{2}}$ orbital with the original localized hole, the already weak AF coupling is further suppressed and hence the superconductivity is weakened. The critical temperature should also be sensitive to the doping level. In addition, pseudogap should emerge at low temperature as in cuprates. On the contrary, the doped holes on the $d_{x y}$ or/and $d_{3 z^{2}-r^{2}}$ orbital may enhance the original $\mathrm{AF}$ couplings although the average spin of a carrier is very weak. In addition, the $\mathrm{SC}$ critical temperature depending on the Hund coupling $J_{h}$ and the spin fluctuation $\chi_{d}$ is not directly related to the doping level unless the carrier density is too low. It is not likely to form a pseudogap due to the ferromagnetic coupling between the carriers and the local magnetic moments in our model. We expect more experiments to check these differences.

The Nd $5 d$ electrons have been ignored in our model for the doped holes dominate the transport and thermodynamics at low temperature in the high doped examples. Nevertheless, in the recent paper 65, it was found that the Hall coefficients become negative at the doping $x$ below 0.175 , which means that electrons may be the dominant carriers at low doping. The $5 d$ electron as carrier could couple to the Ni localized $d_{x^{2}-y^{2}}$ magnetic moments via the Kondo interaction [36]. Thus, the interaction between the $5 d$ electrons and spin fluctuation in nickelates could be described by the Kondo-Heisenberg model [46]. However, since the Kondo coupling $\left(J_{K} \sim 0.1\right.$ $\mathrm{eV})$ is around ten times smaller than the Hund coupling $\left(J_{h} \sim 1 \mathrm{eV}\right)$, the $5 d$ electrons give much weaker contribution to the superconductivity, and their selfenergy renormalization in the normal state is also much weaker than that of the $3 d$ holes, namely, $T_{c} \sim \omega_{c} e^{-1 / \lambda}$ with $\lambda \sim J_{K}^{2}, J_{h}^{2}$ and $\operatorname{Im} \Sigma(\omega, T) \sim J_{K}^{2}, J_{h}^{2}$, respectively.

We have assumed that the momentum-integral spinfluctuation spectra of localized Ni localized $d_{x^{2}-y^{2}}$ holes on the Ni-O planes take the similar form of the underdoped cuprates, and then the marginal Fermi liquid-like self-energy is obtained. We expect that the neutron scattering and more transport experiments could be conducted to verify our assumption. Moreover, if the doped holes enter the $d_{x y}$ or/and $d_{3 z^{2}-r^{2}}$ orbital then the doping does not suppress the AF fluctuations. This also could be judged by the neutron scattering measurements. In addition, to experimentally determine if the doped holes enter the $d_{x y}$ or/and $d_{3 z^{2}-r^{2}}$ orbital, one way is to apply Ni L-edge polarized x-ray absorption near edge structure (XANES) on the single-crystals to study the distribution of holes in the Ni $3 d$ orbitals [66].

In conclusion, we have proposed a Hund-Heisenberg model to investigate the unconventional $\mathrm{SC}$ in the infinite-layer nickelates superconductor. By analyzing the transport experiments, we suggest that the doped holes enter the $\mathrm{Ni} d_{x y}$ or/and $d_{3 z^{2}-r^{2}}$ orbitals, and form a conducting band. The doped holes interact with the localized holes on $d_{x^{2}-y^{2}}$ orbital through the onsite Hund coupling. We show that the non-Fermi liquid state in normal phase results from the carrier gas interacting with the spin fluctuations of the localized holes. In the superconducting phase, it is still the short-range spin fluctuations that mediate the carriers into Cooper pairs and leads to $d$-wave superconductivity. We expect experiments to check our predictions that the doped holes slightly enhance the spin fluctuations and a pseudogap hardly forms in nickelates. We have provided a new SC mechanism for multi-orbital strongly correlated systems, e.g. iron pnictides and it should aid in probing or synthesizing new superconductors in transition or rare-earth metal oxides.

Acknowledgements - We are thankful to Xun-Wang Yan, Yuehua Su and Myung Joon Han for fruitful discussions. This work is supported by the National Natural Science Foundation of China (91750111, 11874188, U1930401 and 11874075), and National Key Research and Development Program of China (2018YFA0305703) and Science Challenge Project (TZ2016001). 
[1] J. G. Bednorz and K. A. Müller, Z. Phys. B 64, 189 (1986).

[2] F. Steglich, J. Aarts, C. D. Bredl, W. Lieke, D. Meschede, W. Franz, and H. Schäfer, Phys. Rev. Lett. 43, 1892 (1979).

[3] P. A. Lee, N. Nagaosa, and X.-G. Wen, Rev. Mod. Phys. 78, 17 (2006).

[4] Y. Kamihara, T. Watanabe, M. Hirano, and H. Hosono, J. Am. Chem. Soc. 130, 3296 (2008).

[5] Y. Maeno, H. Hashimoto, K. Yoshida, S. Nishizaki, T. Fujita, J. Bednorz, and F. Lichtenberg, Nature 372, 532 (1994).

[6] D. Li, K. Lee, B. Y. Wang, M. Osada, S. Crossley, H. R. Lee, Y. Cui, Y. Hikita, and H. Y. Hwang, Nature 572, 624 (2019).

[7] V. I. Anisimov, D. Bukhvalov, and T. M. Rice, Phys. Rev. B 59, 7901 (1999).

[8] K.-W. Lee and W. E. Pickett, Phys. Rev. B 70, 165109 (2004).

[9] Y. J. Yan, M. Q. Ren, H. C. Xu, B. P. Xie, R. Tao, H. Y. Choi, N. Lee, Y. J. Choi, T. Zhang, and D. L. Feng, Phys. Rev. X 5, 041018 (2015).

[10] Y. K. Kim, N. Sung, J. Denlinger, and B. Kim, Nature Phys. 12, 37 (2016).

[11] J. Chaloupka and G. Khaliullin, Phys. Rev. Lett. 100, 016404 (2008).

[12] U. Schwingenschlögl, C. Schuster, and R. Frésard, Annalen der Physik 18, 107 (2009).

[13] P. Hansmann, X. Yang, A. Toschi, G. Khaliullin, O. K. Andersen, and K. Held, Phys. Rev. Lett. 103, 016401 (2009).

[14] A. Ikeda, Y. Krockenberger, H. Irie, M. Naito, and H. Yamamoto, Appl. Phys. Express 9, 061101 (2016).

[15] M. Kawai, S. Inoue, M. Mizumaki, N. Kawamura, N. Ichikawa, and Y. Shimakawa, Appl. Phys. Lett. 94, 082102 (2009).

[16] D. Kaneko, K. Yamagishi, A. Tsukada, T. Manabe, and M. Naito, Physica C: Superconductivity 469, 936 (2009).

[17] S. Ryee, H. Yoon, T. J. Kim, M. Y. Jeong, and M. J. Han, Phys. Rev. B 101, 064513 (2020).

[18] J. Hirsch and F. Marsiglio, Physica C (Amsterdam) 566, 1353534 (2019), ISSN 0921-4534.

[19] A. S. Botana and M. R. Norman, Phys. Rev. X 10, 11024 (2020).

[20] M. Hayward, M. Green, M. Rosseinsky, and J. Sloan, J. Am. Chem. Soc. 121, 8843 (1999).

[21] M. Hepting, D. Li, C. J. Jia, H. Lu, E. Paris, Y. Tseng, X. Feng, M. Osada, E. Been, Y. Hikita, et al., Nat. Mater. 19, 381 (2020).

[22] H. Sakakibara, H. Usui, K. Suzuki, T. Kotani, H. Aoki, and K. Kuroki, arXiv:1909.00060 (2019).

[23] J. Gao, Z. Wang, C. Fang, and H. Weng, arXiv:1909.04657 (2019).

[24] F. Bernardini, V. Olevano, and A. Cano, Phys. Rev. Ressearch 2, 013219 (2020).

[25] P. Jiang, L. Si, Z. Liao, and Z. Zhong, Phys. Rev. B 100, 201106(R) (2019).

[26] M. Hayward and M. Rosseinsky, Solid State Sci. 5, 839 (2003)

[27] H. Zhang, L. Jin, S. Wang, B. Xi, X. Shi, F. Ye, and J.-W. Mei, Phys. Rev. Research 2, 013214 (2020).
[28] X. Wu, D. D. Sante, T. Schwemmer, W. Hanke, H. Y. Hwang, S. Raghu, and R. Thomale, Phys. Rev. B 101, 060504 (2020).

[29] Y. Nomura, M. Hirayama, T. Tadano, Y. Yoshimoto, K. Nakamura, and R. Arita, Phys. Rev. B 100, 205138 (2019).

[30] N. Singh, arXiv:1909.07688 (2019).

[31] M.-Y. Choi, K.-W. Lee, and W. E. Pickett, Phys. Rev. B 101, 020503 (2020).

[32] M. Jiang, M. Berciu, and G. A. Sawatzky, Phys. Rev. Lett. 124, 207004 (2020).

[33] Y.-H. Zhang and A. Vishwanath, arXiv:1909.12865 (2019)

[34] L.-H. Hu and C. Wu, Phys. Rev. Research 1, 032046 (2019).

[35] P. Werner and S. Hoshino, Phys. Rev. B 101, 041104 (2020).

[36] G.-M. Zhang, Y.-F. Yang, and F.-C. Zhang, Phys. Rev. B 101, 020501 (2020).

[37] Y. Fu, L. Wang, H. Cheng, S. Pei, X. Zhou, J. Chen, S. Wang, R. Zhao, W. Jiang, C. Liu, et al., arXiv:1911.03177 (2019).

[38] Q. Li, C. He, J. Si, X. Zhu, Y. Zhang, and H.-H. Wen, Nat. Commun. 1, 1 (2020).

[39] X. Zhou, Z. Feng, P. Qin, H. Yan, S. Hu, H. Guo, X. Wang, H. Wu, X. Zhang, H. Chen, et al., Rare Met. 39, 368 (2020).

[40] L. Si, W. Xiao, J. Kaufmann, J. M. Tomczak, Y. Lu, Z. Zhong, and K. Held, arXiv:1911.06917 (2019).

[41] F. Lechermann, Phys. Rev. B 101, 081110 (2020).

[42] T.-H. Lee, A. Chubukov, H. Miao, and G. Kotliar, Phys. Rev. Lett. 121, 187003 (2018).

[43] A. Georges, L. d. Medici, and J. Mravlje, Annual Review of Condensed Matter Physics 4, 137 (2013).

[44] K. Haule and G. Kotliar, New Journal of Physics 11, 025021 (2009).

[45] P. Werner, E. Gull, M. Troyer, and A. J. Millis, Phys. Rev. Lett. 101, 166405 (2008).

[46] J. Chang and J. Zhao, Eur. Phys. J. B 90, 154 (2017).

[47] J. Zaanen and A. M. Oleś, Phys. Rev. B 37, 9423 (1988).

[48] S. M. Hayden, G. Aeppli, H. Mook, D. Rytz, M. F. Hundley, and Z. Fisk, Phys. Rev. Lett. 66, 821 (1991).

[49] B. Keimer, R. J. Birgeneau, A. Cassanho, Y. Endoh, R. W. Erwin, M. A. Kastner, and G. Shirane, Phys. Rev. Lett. 67, 1930 (1991).

[50] J. M. Tranquada, P. M. Gehring, G. Shirane, S. Shamoto, and M. Sato, Phys. Rev. B 46, 5561 (1992).

[51] F. C. Zhang and T. M. Rice, Phys. Rev. B 37, 3759 (1988).

[52] P. Coleman, in Handbook of Magnetism and Advanced Magnetic Materials, edited by $\mathrm{H}$. Kronmüller and S. Parkin (John Wiley and Sons, New York, 2007), vol. 1, pp. $95-148$.

[53] D. J. Scalapino, E. Loh, and J. E. Hirsch, Phys. Rev. B 34, 8190 (1986).

[54] N. Bickers, D. Scalapino, and R. Scalettar, Int. J. Mod. Phys. B 01, 687 (1987).

[55] M. Inui, S. Doniach, P. J. Hirschfeld, and A. E. Ruckenstein, Phys. Rev. B 37, 2320 (1988).

[56] C. Dong, J. K. Liang, G. C. Che, S. S. Xie, Z. X. Zhao, Q. S. Yang, Y. M. Ni, and G. R. Liu, Phys. Rev. B 37, 
$5182(1988)$.

[57] G. Kotliar and J. Liu, Phys. Rev. B 38, 5142 (1988).

[58] P. Monthoux, A. V. Balatsky, and D. Pines, Phys. Rev. Lett. 67, 3448 (1991).

[59] T. Moriya, Y. Takahashi, and K. Ueda, J. Phys. Soc. Jpn. 59, 2905 (1990).

[60] A. J. Millis, H. Monien, and D. Pines, Phys. Rev. B 42, 167 (1990).

[61] A. Chubukov, D. Pines, and J. Schmalian, in Superconductivity, edited by K. Bennemann and J. Ketterson (Springer Berlin Heidelberg, 2008), pp. 1349-1413.

[62] D. J. Scalapino, Rev. Mod. Phys. 84, 1383 (2012).
[63] J. Böker, M. A. Sulangi, A. Akbari, J. C. S. Davis, P. J. Hirschfeld, and I. M. Eremin, arXiv:2004.02768 (2020).

[64] Y. Gu, S. Zhu, X. Wang, J. Hu, and H. Chen, arXiv:1911.00814 (2019).

[65] D. Li, B. Y. Wang, K. Lee, S. P. Harvey, M. Osada, B. H. Goodge, L. F. Kourkoutis, and H. Y. Hwang, arXiv:2003.08506 (2020).

[66] G. Kaindl, O. Strebel, A. Kolodziejczyk, W. Schäfer, R. Kiemel, S. Lösch, S. Kemmler-Sack, R. Hoppe, H. Müller, and D. Kissel, Physica B: Condensed Matter 158, 446 (1989). 